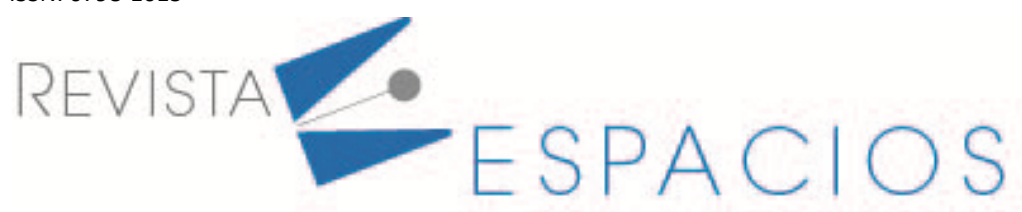

\title{
NIC 2 y su efecto en la presentación razonable de los Estados Financieros de la Empresa Macoser S.A. (Ecuador)
}

\section{IAS 2 and its effect on the fair presentation of Financial States of the Company Macoser S.A.}

\author{
ESCANDÓN, Ruth ${ }^{1}$ \\ GUERRERO, Jenifer ${ }^{2}$ \\ CAMPOS, Hugo F. ${ }^{3}$ \\ ESPINOZA, Rosa A. ${ }^{4}$ \\ ESPINOZA, Wilmer ${ }^{5}$
}

\begin{abstract}
Resumen
Los inventarios representan activos de gran valor para la empresa, actualmente existen problemas en el manejo de estos y no aplica lo dispuesto en la Norma Internacional №. 2 sobre Inventarios. El objetivo es aplicar la NIC 2 de forma correcta con el fin de medir su impacto en los Estados Financieros. La investigación es de enfoque cualitativo y diseño descriptivo - explicativo, obteniendo como resultado una propuesta basada en la normativa actual, que contribuya a sincerar los saldos encaminados al tratamiento contable.
\end{abstract}

Palabras claves: inventarios, nic 2, ajustes, valor neto realizado

\begin{abstract}
Inventories represent assets of great value to the company; currently there are problems in the management thereof and the provisions of International Standard No. 2 on Inventories. The objective is to apply IAS 2 correctly so as to measure the impact on the Financial Statements. The research is of a qualitative approach and descriptive - explanatory design, allowed to reach conclusions where a proposal based on current regulations is proposed, which helps to clarify the balances for accounting treatment.

Key words: inventories, ias 2, adjustments, net realized value
\end{abstract}

\section{Introducción}

La empresa Macoser S.A es una empresa familiar que tiene como objetivo principal la comercialización, importación, distribución y ventas de línea textil y líneas de productos tecnológicos, ejerce actividades desde hace 24 años en el mercado ecuatoriano, es distribuidor autorizado de marcas reconocidas y se encuentra

\footnotetext{
1 Universidad Laica Vicente Rocafuerte de Guayaquil. Egresado. E-mail: ruth221193@hotmail.com.

2 Universidad Laica Vicente Rocafuerte de Guayaquil. Egresado. E-mail: : jeniferguerrero87@gmail.com

${ }^{3}$ Facultad Ciencias Administrativas. Docente Tiempo Completo, Universidad Técnica de Babahoyo. Docente medio tiempo, Universidad Laica Vicente Rocafuerte de Guayaquil. Contador Público Autorizado. Magíster en Administración y Dirección de Empresas, MsC. en Educación. E-mail: hcampos@utb.edu.ec

${ }^{4}$ Universidad Estatal de Milagro. Docente tiempo completo. Economista, Magíster en Administración y Dirección de Empresas.

PhD. en Administraciòn . E-mail: respinozat@unemi.edu.ec

${ }^{5}$ Docente Tiempo Completo, Universidad Técnica de Babahoyo, Docente tiempo parcial, Universidad Laica Vicente Rocafuerte de Guayaquil. Contador Público Autorizado. Magíster en Tributación. .E-mail: wilmerespinozat6@hotmail.com.
} 
ubicada en el centro de Guayaquil. En virtud del crecimiento de la empresa en el transcurso de los años, se han originado situaciones que afectan el normal desarrollo de actividades de control y planificación de los inventarios; en consecuencia los datos que se presentan en los Estados Financieros no se ajustan a la realidad, los inventarios y bajas de inventario no se registran de manera oportuna, lo cual se traduce en montos y números irreales que perjudican el proceso de toma de decisiones por parte de la gerencia de la empresa en relación con la gestión del inventario de mercancías. El control en las entradas y salidas, almacenamiento, y conteo de los productos en existencia son parte también de los problemas que enfrenta actualmente la empresa. La pregunta que se planteó fue el inicio de un estudio que se basó en el siguiente problema: ¿Qué impacto tendría la correcta aplicación de la Norma Internacional de Contabilidad 2-Inventarios en los Estados Financieros (NIC 2) , de la empresa Macoser S.A.? El problema formulado requirió el cumplimiento del siguiente objetivo investigativo: Aplicar la NIC 2 de forma correcta con el fin de medir el impacto en los Estados Financieros de la empresa Macoser S.A., siendo una alternativa de solución para reconocer el costo real de la mercadería almacenada y de poca rotación, determinando el costo y su registro como gastos, incluyendo los ajustes para reflejar el Valor Neto Realizable (VNR), considerando lo que estipula la NIC 2, referida a los inventarios.

\subsection{Concepto de Inventario}

"La norma internacional contable 2 (NIC 2) define a los inventarios como activos poseídos para ser vendidos en el curso normal de explotación en proceso de producción de cara a esa venta o en forma de materiales o suministros para ser consumidos en el proceso de producción y los necesarios para llevar a cabo la venta." (Hansen, 2009, p.251). Esta norma establece la información que debe ser presentada en los estados financieros, con la finalidad de mejorar y armonizar las prácticas contables.

Básicamente, los inventarios se pueden definir como el conjunto de artículos o productos que se acopian en el almacén pendientes de ser manipulados en el proceso productivo o mercantilizados. Otra definición de inventario vinculada al ámbito económico es la correlación ordenada de bienes de una organización o persona, en la que además de los stocks, se incluyen también otra clase de bienes. También el documento que acumula la relación de dicho artículo se le conoce como inventario. (Economiasimple, 2014, p.1). Los inventarios representan los activos de una empresa, y por lo tanto ameritan resguardo, control, registro, y organización de acuerdo con las exigencias de las Normas Internacionales de Contabilidad.

Para Castillo (2013), la adopción de la NIC 2, comprende un efecto de relativos, evitando la omisión de partidas que puedan afectar de forma sustancial la información financiera que sirve de base en la toma de decisiones administrativas de la empresa, siendo los inventarios la principal fuente de ingresos de la empresa. Sin embargo, la aplicación de esta norma permite una correcta valuación de sus costos mediante un buen control, y una adecuada presentación en los estados financieros. Además, al coordinar todas las actividades de la empresa se logra un mejor resultado de gestión.

\subsection{Administración de Inventarios}

Es la diligencia de instrucciones y métodos que tienen por centro instaurar, poner en efecto y mantener las cantidades más favorables de materias primas, producción en proceso, artículos terminados y otros inventarios, mermando los costos a que den lugar, para aportar y lograr los fines de la organización (UAL, 2013). La gerencia de una empresa debe tener claro que el control adecuado de los inventarios generará beneficios para la organización, incluyendo el logro de metas y objetivos de rentabilidad que se plantee. Si se descuida el correcto manejo y registro de las entradas y salidas de los inventarios, las consecuencias suelen ser muy negativas para la gestión. 
Según Ferrari (2018), un inventario por encima del nivel máximo óptimo incrementa los costos, representa una enorme suma de capital inmovilizado en deterioro de la eficiencia, y trae consigo riesgos de caducidad u obsolescencia. Por el contrario, un inventario por debajo del nivel mínimo óptimo puede traer como consecuencia escasez de productos que implicarán costos y logística innecesarios, parada en los procesos o clientes insatisfechos. Un nivel de inventario óptimo es aquel que compensa costos y riesgos por exceso, así como costos y riesgos por déficit de este.

Para lograr niveles óptimos de inventarios en mercancías, a un mínimo costo, así como su adecuado control y trazabilidad, existen los sistemas de administración de inventarios. Para adoptar la norma internacional en el tratamiento contable de los Inventarios fue necesario definir el marco conceptual específico de la empresa, que se describe a continuación, con el propósito de proporcionar la aplicación y análisis de los resultados.

A continuación se describe el marco conceptual específico de la empresa:

- Almacén: Local, edificio o parte de éste acondicionado para depositar o guardar gran cantidad de artículos, productos o mercancías para su posterior venta, uso o distribución (Ferrari, 2018).

- Costos de Importación: Comprende el importe original de adquisición más todos los costes para poner el producto importado en la bodega de la empresa.

- Estados Financieros: Los Estados Financieros constituyen una representación estructurada de la situación financiera y del rendimiento financiero de la entidad (NIC2, 2005).

- Existencias: Las existencias constituyen el realizable de explotación, y está integrado por los stocks que la empresa necesita mantener para desarrollar su actividad productiva y comercial (Rajadel, Trullàs, \& Simo, 2014).

- Importación: Representa el conjunto de bienes y servicios comprados por un país en territorio extranjero para su utilización en territorio nacional.

- Stock: Es un abastecimiento de artículos en espera de ser utilizado posteriormente, con el objetivo de disponer de la cantidad necesaria, en el momento oportuno, en el lugar preciso con el mismo costo.

- Valor Neto Realizable: Es el precio estimado de venta de un activo en el curso normal de la explotación, menos los costes estimados para terminar su producción y los necesarios para llevar a cabo la venta (NIC2, 2005).

- Valor Razonable: Es el importe por el cual puede ser intercambiado un activo o cancelado un pasivo, entre partes interesadas y debidamente informadas, que realizan una transacción en condiciones de independencia mutua (NIC2, 2005).

\section{Metodología}

La investigación se realizó bajo un enfoque cualitativo y cuantitativo (mixto), con un alcance exploratorio y descriptivo, se recogió información documental, bibliográfica y a través de la aplicación de instrumentos de recolección de información.

El tipo investigación es descriptivo porque ha permitido describir la realidad de la situación de la empresa y analizar e identificar los distintos procedimientos y factores que se dan entorno al problema identificado. 
La investigación descriptiva "presupone que el investigador tiene conocimiento previo sobre el problema a o la situación en cuestión. Por tanto, a diferencia de la investigación exploratoria, los estudios de investigación se llevan a cabo después que el investigador haya adquirido una comprensión firme de la situación" (Rosendo, 2018, p. 1).

La investigación tiene un enfoque cuantitativo, porque a través del cual se analiza la presentación de los Estados Financieros en referencia a la cuenta de inventarios. Según Hernández, Fernández, \& Baptista (2014), manifiestan que "se utiliza la recolección de datos para probar hipótesis con base en la medición numérica y el análisis estadístico, con el fin establecer pautas de comportamiento y probar teorías". De esta manera, se utilizan distintos instrumentos para recolectar toda la información pertinente y necesaria para complementar la investigación como la entrevista y encuestas.

Entrevista: Se refiere al "ejercicio de desplegar una charla con una o más personas con el objeto de conversar sobre determinados temas y con un fin definitivo" (Pérez \& Gardey, 2012, p.1). La entrevista permite establecer un contacto directo con los directivos de la empresa, y obtener información confiable relacionada al tema.

Encuesta: Es una técnica común y ampliamente utilizada en trabajos de investigación para obtener y elaborar datos de manera rápida y eficaz. En el presente estudio se aplica encuesta al personal operativo que labora en la empresa MACOSER S.A. cuyos resultados fueron tabulados y graficados para una mejor comprensión de los datos obtenidos y se procedió hacer el análisis comparativo de los estados financieros de los años 2016 y 2017 de la empresa, así como también información relacionada con el stock en inventario y la mercancía de la que se dispone al 31/12/2017.

La población está representada por el personal que labora en la empresa MACOSER S.A. número que asciende a la cantidad de 45 empleados, distribuidos en los distintos departamentos que conforman la estructura organizativa.

Tabla 1

Población de Estudio

\begin{tabular}{lc}
\hline Población & Cantidad \\
\hline Bodega & 8 \\
Logística & 1 \\
Contabilidad & 4 \\
Asistente de Gerencia & 1 \\
Vendedores & 31 \\
Total & $\mathbf{4 5}$ \\
\hline
\end{tabular}

Fuente: Empresa MACOSER S.A. (2018)

Elaborado por: Los autores

\subsection{Técnicas de Investigación}

Entrevista: Se aplicó la entrevista para obtener información desde las personas que se encuentran detrás de los procesos de la gestión del inventario, con el fin de levantar información necesaria que permita analizar el contexto en el cual estas personas ejecutan sus actividades.

La población está representada por el personal vinculado al área administrativa: 
Tabla 2

Población de Estudio

\begin{tabular}{lc}
\hline Población & Cantidad \\
\hline Contabilidad & 1 \\
Compras & 1 \\
Jefe de Bodega & 1 \\
Total & $\mathbf{3}$ \\
\hline
\end{tabular}

Fuente: Empresa Inplasban S.A. (2018)

Elaborado por: Los autores

\section{Resultados}

Para realizar la investigación a través de la aplicación de las entrevistas y encuestas, la población y muestra abarcó la totalidad de los empleados, y se toma como referente una pregunta de la encuesta realizada a los 45 empleados que representa la muestra y la entrevista realizada al personal vinculado al área administrativa, las mismas que se presentan a continuación:

Se realiza la entrevista aplicando la técnica de focus group al personal del area administrativa, una de las preguntas seleccionadas es ¿La empresa aplica lo estipulado en la NIC 2 para el registro y control de los inventarios? , Respondiendo que la empresa no aplica lo estipulado en la NIC 2 para llevar un correcto control de la cuenta de inventario. Es importante conocer que La NIC2 suministra una guía práctica para la determinar el costo de los inventarios, permitiendo realizar los ajustes y registros correctamente.

A la segunda pregunta sobre si ¿Actualmente los Estados Financieros reflejan saldo real en las cuentas del inventario?, alegaron que actualmente los Estados Financieros no reflejan el saldo real en las cuentas del inventario. Un Estado Financiero que no refleje el saldo real en las cuentas incumple los Principios Contables, tales como revelación suficiente, de registro, y valor de adquisición.

En la encuesta se toma como referente una de las preguntas sobre el control de investario, que se presenta acontinuación.

¿La empresa realiza con frecuencia el conteo físico del inventario?

Tabla 3

\begin{tabular}{ccc}
\multicolumn{3}{c}{ Conteo físico del inventario } \\
\hline Descripción & Frecuencia & Porcentaje \\
\hline Si & 19 & $42 \%$ \\
No & 26 & $58 \%$ \\
Totales & 45 & $100 \%$ \\
\hline
\end{tabular}

Fuente: Encuesta aplicada a personal de la empresa

De acuerdo a los resultados obtenidos en la encuesta, se puede observar que un $58 \%$ de los empleados encuestados opina que la empresa no realiza conteo físico del inventario, y un $42 \%$ indica que si se realiza. El conteo físico es un proceso de gran importancia que permite a la gerencia conocer de manera oportuna la cantidad de mercancía o equipos con los que se dispone.

\subsection{Análisis de los resultados obtenidos}

De acuerdo a los resultados obtenidos, la empresa no lleva el control del inventario de manera adecuada, ni se aplica la NIC 2 en los procedimientos de registro y control. Existe mercancía en bodega que no está apta para la venta, esto incide negativamente en los saldos presentados en los Estados Financieros, se muestra un activo que en su totalidad no va a generar los ingresos previstos, según saldos en cuentas. 
De manera periódica no se realiza un conteo físico del inventario, lo cual afecta el control y se desconoce con exactitud de cuanto se dispone en los almacenes. De igual manera se logró conocer a través de las respuestas dadas por los encuestados, la inexistencia de una planificación adecuada para la compra de la mercancía, es decir, no existe un control de compras riguroso que contribuya a minimizar costos y gastos innecesarios.

Según las respuestas dadas por el personal que labora en el Departamento de Contabilidad, la empresa no aplica la Norma Internacional Contable Nro. 2, lo que afecta el tratamiento contable de los inventarios, porque se mantiene en las cuentas montos significativos de inventarios que ajustados a la realidad pueden disminuir las expectativas de ventas.

De acuerdo a lo señalado anteriormente es necesario realizar un ajuste en la cuenta de inventario, considerada relevante para determinar la liquidez de la empresa. Además del ajuste, el dar de baja a los productos que no están aptos para la venta permitirá conocer el inventario disponible y su valor correcto en una fecha determinada.

\subsection{Análisis comparativo de los Estados Financieros de los años 2016-2017}

A través de la información recolectada en las entrevistas y encuestas realizadas, así como los análisis realizados a los Estados Financieros de la Empresa Macoser S.A., correspondientes a los años 2016 y 2017, se pudo determinar que la empresa no cumple con lo dispuesto en la NIC 2, tampoco mantiene los saldos ajustados al valor real de los activos y no ha desincorporado mercancía no apta para la venta lo que aumenta el valor de los activos de manera errada.

Tabla 4

Inventarios de Productos terminados y mercadería

\begin{tabular}{ccc}
\hline & \multicolumn{2}{c}{$\begin{array}{c}\text { Inventarios de Productos terminados y mercad. En Almacén } \\
\text { (Excluyendo obras/inmuebles terminados para la venta) }\end{array}$} \\
\hline Año 2016 & $\$$ & $5.271 .396,66$ \\
Año 2017 & $\$$ & $5.109 .462,45$ \\
Variación & $\$$ & $\mathbf{1 6 1 . 9 3 4 , 2 1}$ \\
\hline
\end{tabular}

Fuente: Estado de Resultado Integral Empresa Macoser S.A.

Según información cotejada en los Estados Financieros correspondientes a los años 2016 y 2017 de la Empresa Macoser S.A. se observa una variación en el monto de los Inventarios de Productos por la cantidad de \$ $161.934,21$ representando una leve disminución de un 3,07\% con respecto al año 2016 . Esto a su vez indica que la empresa mantiene en existencia menor cantidad de mercancía con respecto al año anterior resultado del aumento en las ventas. Esta variación puede observarse en el Ventas realizadas en el año 2017 con respecto al año 2016, montos que se encuentran reflejados en los Estados de Resultado Integral de los correspondientes años.

\subsection{Variación en el Costo de Ventas para los años 2016 y 2017}

El Costo de Ventas de la empresa MACOSER S.A. está representado por las compras realizadas durante el ejercicio económico más el inventario inicial menos el inventario final, el cual disminuye para el año 2017 en un 3,07\% respecto al año 2016. 
Tabla 5

Variación en el Costo de Ventas 2016-2017

\begin{tabular}{|c|c|c|}
\hline & AÑO 2016 & AÑO 2017 \\
\hline COSTO DE VENTAS & $\$ 4.006 .011,05$ & $\$ 4.372 .266,29$ \\
\hline $\begin{array}{l}\text { Inventario Inicial de Bienes no producido por el sujeto } \\
\text { pasivo }\end{array}$ & $\$ 5.896 .348,06$ & $\$ 5.271 .396,66$ \\
\hline $\begin{array}{l}\text { Compra de materiales de bienes no producidos por el } \\
\text { sujeto pasivo }\end{array}$ & $\$ 168.550,50$ & $155.195,01$ \\
\hline Importación de bienes no producidos por el sujeto pasivo & $\$ 3.212 .509,15$ & $\$ 4.055 .137,07$ \\
\hline $\begin{array}{l}\text { Menos: Inventario final de bienes no producidos por el } \\
\text { sujeto pasivo }\end{array}$ & $\$ 5.271 .396,66$ & $\$ 5.109 .462,45$ \\
\hline
\end{tabular}

Fuente: Estado de Resultado Integral Empresa Macoser S.A.

La Cuenta Importación de bienes no producidos por el sujeto pasivo muestra un aumento significativo para el año 2017 de un 26\%. Es importante señalar que estos productos adquiridos a través de importación aumentan su costo de adquisición debido a los aranceles y fletes que deben ser cancelados para el ingreso legal de la mercancía al país, por lo que a su vez el precio de venta se incrementa. Según información suministrada por la Gerencia de la empresa, se cancelan los siguientes costos de adquisición del producto, lo que incrementa el valor de la mercancía:

Tabla 6

Costos de adquisición

COSTOS DERIVADOS DE ADQUISICION

\begin{tabular}{l} 
Seguro 6\% \\
Almacenamiento 14\% \\
Custodia 6\% \\
Agente de Aduana 21\% \\
Fodinfa $1 \%$ \\
Arancel 25\% \\
Flete $10 \%$ \\
\hline \multicolumn{1}{c}{ Elaborado por: los autores }
\end{tabular}

\subsubsection{Costo Real del Inventario}

Se obtuvo información de los saldos en la cuenta de inventario al 31/12/2016 y 31/12/2017, a través de los Estados Financieros facilitados por la Empresa. El monto total obtenido representa toda la cantidad de mercancía en existencia, la clasificación de la mercancía supera los mil ítems, razón por la cual se ha tomado la decisión de apuntar la investigación hacia la mercancía relacionada con las máquinas de coser en las distintas presentaciones y marcas que tiene la empresa para la venta, por ser de gran valor y su vez forma parte de los principales productos relacionados con la actividad de la empresa, tales como: Máquinas de coser, bordadoras, botoneras, máquinas marca brother, ojaladoras, overlock, recubridoras, ribeteadoras y zigzag, entre otros.

\subsubsection{Valor Neto de Realización (VNR)}

El Valor Neto de Realización está representado por el precio estimado de venta de un activo menos los costos estimados para terminar su producción, menos los costos de venta. Considerando la actividad principal de la Empresa Macoser S.A., no se aplican costos estimados para terminar la producción porque la empresa solo se encarga de la comercialización de productos ya terminados, por lo tanto, solo se disminuye el Costo de Ventas.

Según la NIC 2 Existencia, indica los inventarios registrados a costo historicos, deben ser valuados y valorados de acuerdo a valor del mercado, el más bajo, para lo cual se utiliza la técnica del valor de realización, lo que 
resulta apropiado agrupar partidas similares para el ajuste de los inventarios. En el presente caso se utiliza una misma línea de productos cuyos propósitos y usos finales son afines, así como también se distribuyen y venden en el mismo espacio geográfico.

El Valor Neto Realizable se calcula tomando en consideración información confiable suministrada por la empresa, sobre el importe del que se dispone en los saldos contables, y se cogen como base los datos expuestos en los Estados Financieros de los años 2016 y 2017.

\subsubsection{Cálculo del Valor Neto Realizable}

Tabla 7

Cálculo del Valor Neto Realizable (VNR)

\begin{tabular}{|c|c|c|c|c|c|}
\hline \multicolumn{6}{|c|}{ Expresado en dólares } \\
\hline $\begin{array}{c}\text { Total Valor en } \\
\text { Libros } \\
\text { (Unitario) }\end{array}$ & $\begin{array}{c}\text { Precio Venta } \\
\text { Estimado } \\
\text { (Unitario) }\end{array}$ & $\begin{array}{l}\text { Precio de Venta } \\
\text { Estimado (Total) }\end{array}$ & $\begin{array}{c}\text { Gasto de Ventas } \\
\text { Total } 22 \%\end{array}$ & $\begin{array}{c}\text { Gasto de } \\
\text { Distribución } \\
\text { Total } 8 \%\end{array}$ & $\begin{array}{c}\text { Valor Neto } \\
\text { Realizable Total }\end{array}$ \\
\hline $3.229 .808,80$ & $653.783,73$ & $4.621 .374,70$ & $1.016 .702,43$ & $369.709,98$ & $3.234 .962,29$ \\
\hline
\end{tabular}

De acuerdo a los resultados que se observan en la tabla №. 7, es necesario que la Empresa MACOSER S.A. realice un reconocimiento y medición de los inventarios al valor neto realizable, para lo cual se ha comparado el valor en libros que actualmente la empresa mantiene en Inventario y el cálculo del costo de adquisición y de ventas por cada uno de los productos de la línea textil. En un primer análisis se observa que el valor neto realizable obtenido está por encima del valor en libros, por lo tanto no existe ajuste a los inventarios .

Si el Valor Neto Realizable arroja un valor por debajo del valor en libros, la empresa estará sufriendo una pérdida en el inventario, que le conlleva a realizar el ajuste correspondiente. Esto a su vez proporciona a la empresa datos más reales de lo que puede disponer como ingreso a futuro, cumpliendo con lo que establece la Norma Internacional Contable Nro. 2.

A continuación, se muestra tabla №. 8 el cálculo del ajuste del inventario textil al Valor Neto Realizable, arrojando las siguientes diferencias:

Tabla 8

Ajuste del Inventario Textil al Valor Neto Realizable

\begin{tabular}{cccccccc}
\hline \multicolumn{10}{c}{ Expresado en Dólares } \\
\hline $\begin{array}{c}\text { Unida } \\
\text { des }\end{array}$ & $\begin{array}{c}\text { Costo } \\
\text { Unitario }\end{array}$ & $\begin{array}{c}\text { Total Valor En } \\
\text { Libros } \\
\text { (Unitario) }\end{array}$ & $\begin{array}{c}\text { Precio } \\
\text { Venta } \\
\text { Estimado } \\
\text { (Unitario) }\end{array}$ & $\begin{array}{c}\text { Precio De Venta } \\
\text { Estimado (Total) }\end{array}$ & $\begin{array}{c}\text { Gasto De } \\
\text { Ventas Total } \\
22 \%\end{array}$ & $\begin{array}{c}\text { Gasto De } \\
\text { Distribucion } \\
\text { Total }\end{array}$ & $\begin{array}{c}\text { Valor Neto } \\
\text { Realizable } \\
\text { Total }\end{array}$ \\
\hline 953 & $458.899,85$ & $3.229 .808,80$ & $653.783,73$ & $4.621 .374,70$ & $1.016 .702,43$ & $369.709,98$ & $3.234 .962,29$ \\
\hline
\end{tabular}

Elaborado por: los autores (2018)

\subsubsection{Ajuste del Inventario}

Tal como se observa en la Tabla № 9, referida a ajuste del inventario, existe en almacén 179 productos de la línea textil que deben ser ajustados de acuerdo al valor neto realizable obtenido después de disminuir al precio estimado de ventas, los gastos por concepto de distribución y ventas con respecto al valor en libros; el total de la diferencia es de $\$ 31.289,05$. 
Tabla 9

Ajuste del Inventario

\begin{tabular}{clccccc}
\hline Unidades & $\begin{array}{c}\text { TOTAL } \\
\text { VALOR EN } \\
\text { LIBROS }\end{array}$ & $\begin{array}{c}\text { PRECIO DE } \\
\text { VENTA } \\
\text { ESTIMADO } \\
\text { (Total) }\end{array}$ & $\begin{array}{c}\text { GASTO DE } \\
\text { VENTAS } \\
\text { TOTAL 22\% }\end{array}$ & $\begin{array}{c}\text { GASTO DE } \\
\text { DISTRIBUCIO } \\
\text { N TOTAL } \\
8 \%\end{array}$ & $\begin{array}{c}\text { VALOR NETO } \\
\text { RAZONAB. } \\
\text { TOTAL }\end{array}$ & AJUSTE \\
\hline 179 & $\mathbf{8 0 6 . 5 9 9 , 2 5}$ & $\mathbf{1 . 1 0 7 . 5 8 6 , 0 0}$ & $\mathbf{2 4 3 . 6 6 8 , 9 2}$ & $\mathbf{8 8 . 6 0 6 , 8 8}$ & $\mathbf{7 7 5 . 3 1 0 , 2 0}$ & $\mathbf{- 3 1 . 2 8 9 , 0 5}$ \\
\hline
\end{tabular}

Elaborado por: los autores (2018)

\subsubsection{Propuesta de asientos de ajuste a la cuenta de inventario}

Se sugiere ajuste en la cuenta de inventarios, resultado del cálculo del Valor Neto Realizado:

Valor en Libros

$=\$ 806.599,25$

Menos: Valor Neto Realizado

$=(\$ 775.310,20)$

Deterioro en el valor de los inventarios $=\$ 31.289,05$

Registro Asiento Contable al 31/12/2017

\begin{tabular}{|c|c|c|c|}
\hline Fecha & $-1-$ & & \\
\hline \multirow[t]{3}{*}{$31 / 12 / 2017$} & Descripción & Debe & Haber \\
\hline & Pérdida Neta por Deterioro en el Valor de Inventarios & $\$ 31.289,05$ & \\
\hline & $\begin{array}{l}\text { Provisión por valor neto de realización y otras } \\
\text { pérdidas en el Inventario } \\
\text { Para registrar pérdida neta por deterioro en el valor del } \\
\text { inventario, por cálculo del Valor Neto Realizado. }\end{array}$ & & $\$ 31.289,05$ \\
\hline Fecha & $-2-$ & & \\
\hline \multirow[t]{2}{*}{$31 / 12 / 2017$} & Descripción & Debe & Haber \\
\hline & $\begin{array}{l}\begin{array}{l}\text { Provisión por valor neto de realización y otras pérdidas en el } \\
\text { Inventario }\end{array} \\
\qquad \begin{array}{l}\text { Inventario de productos terminados y mercad. En } \\
\text { para la venta) }\end{array} \\
\text { Para crear provisión por valor neto de realización y otras pérdidas } \\
\text { en el inventario al calcular VNR. }\end{array}$ & $\$ 31.289,05$ & $\$ 31.289,05$ \\
\hline
\end{tabular}

Nuevo Valor en Libros de los Inventarios (Por Ajuste al Valor Neto Realizable - Inventario Línea Textil)

Tabla 10

Nuevo Valor en Libros de los Inventarios

\begin{tabular}{ccc}
\hline Unidades & Total Valor En Libros (Unitario) & Precio Venta Estimado (Unitario) \\
\hline 953 & $3.198 .519,76$ & $653.783,73$ \\
\hline
\end{tabular}


Se observa en la Tabla Nro. 10, que una vez ajustado los valores se obtiene un nuevo valor en los libros de la cuenta de inventarios, en el presente caso, el nuevo monto del valor en libros del inventario de mercancía es de \$3.198.519,76 (este monto aún mantiene la mercancía que no está apta para la venta) valor que resulta menor al que inicialmente presentaba la cuenta de $\$ 3.229 .808,80$. Sin embargo, es importante señalar que la empresa debe ajustar aún más su valor en la cuenta de inventario, ya que es necesario considerar disminuir la mercancía que no está apta para la venta y que aumenta el valor del activo, no siendo la realidad con la que cuenta la empresa.

\subsubsection{Ajuste por Deterioro de mercancía no apta para la venta - Línea Textil}

El costo de los inventarios puede que no se recupere, por distintas razones, sin embargo, la norma establece que los activos no deben registrarse en libros por encima de lo que se espera obtener de los mismos, cuando se realice la venta correspondiente. Vale señalar que la empresa mantiene en los libros, monto correspondiente a mercancía que no está apta para la venta, lo cual incrementa el saldo en libros. Con la finalidad de comenzar a sincerar los saldos en cuentas, la empresa realiza un conteo físico al 14/12/2017 (específicamente con la muestra seleccionada) y se logró precisar la mercancía que la empresa no ha desincorporado y aún permanecen en los saldos contables como activos disponibles para la venta. La mercancía clasificada como no apta para la venta, presenta deterioro y averías, fallas de origen, entre otras causas, que impiden la venta normal del producto. Algunos equipos han permanecido en la bodega por más de 5 años y no se han realizado los ajustes correspondientes para disminuir esos valores de los registros contables.

\begin{tabular}{|c|c|c|c|}
\hline Fecha & $-3-$ & & \\
\hline \multirow[t]{3}{*}{$31 / 12 / 2017$} & Descripción & Debe & Haber \\
\hline & Pérdida Neta por Deterioro en el Valor de Inventarios & $\$ 63.509$ & \multirow[b]{2}{*}{$\$ 63.509$} \\
\hline & $\begin{array}{l}\text { Provisión por valor neto de realización y otras } \\
\text { pérdidas en el Inventario } \\
\text { Para registrar pérdida neta por deterioro en el valor del } \\
\text { inventario, resultado de precisar mercancía no apta para la } \\
\text { venta que debe darse de baja. }\end{array}$ & & \\
\hline Fecha & $-4-$ & & \\
\hline \multirow[t]{4}{*}{$31 / 12 / 2017$} & Descripción & Debe & Haber \\
\hline & $\begin{array}{l}\text { Provisión por valor neto de realización y otras pérdidas en el } \\
\text { Inventario }\end{array}$ & $\$ 63.509$ & \multirow{3}{*}{$\$ 63.509$} \\
\hline & $\begin{array}{l}\text { Inventario de productos terminados y mercad. En } \\
\text { Almacén (Excluyendo obras/inmuebles terminados } \\
\text { para la venta) }\end{array}$ & & \\
\hline & $\begin{array}{l}\text { Para registrar provisión por pérdidas en el inventario, resultado de } \\
\text { precisar mercancía no apta para la venta que debe darse de baja. }\end{array}$ & & \\
\hline
\end{tabular}

Elaborado por: los autores (2018)

3.3.7. Nuevo Valor en Libros de los Inventarios (Considerando el Ajuste Valor Neto Realizable y el Ajuste por desincorporación de mercancía no apta para la venta - Línea Textil)

- Valor en Libros Inicial.

$\$ 3.229 .808,80$

Menos:

- Ajuste por Valor Neto Realizable

$\$ 31.289,05$

- Menos: Ajuste por desincorporación de mercancía no apta

para la venta.

$\$ \quad 63.509,00$ 
TOTAL AJUSTE EN EL VALOR DE LOS LIBROS

TOTAL VALOR EN LIBROS DE:

Inventario de Productos Terminados y mercadería en

Almacén - Línea Textil. 3.135.010,75

\subsubsection{Efecto contable en la presentación de los Estados Financieros Ajustados (Año 2017)}

Al disminuir el monto de los activos por la previsión en el cálculo del Valor Razonable Neto y el deterioro por mercancía que no está apta para la venta y a la cual se le debe dar de baja, disminuye en la misma cantidad la utilidad del ejercicio, ya que el monto ajustado forma parte del gastos en el Estado de Resultado Integral. Las pérdidas por deterioro en el valor del inventario ascienden a 529.771,85 con una diferencia de 94.798,05 con respecto al año 2016. Esto incide directamente en las utilidades del ejercicio disminuyéndolas en la misma cantidad.

\subsubsection{Propuesta de implementación del Plan de Cuentas sugerido por la Superintendencia de Compañías del Ecuador}

Se sugiere a la empresa Macoser S.A. aplicar el Plan de Cuentas propuesto por la Superintendencia de Compañías del Ecuador, a fin de que se cumpla con el principio de uniformidad en la información; tal sugerencia se hace en virtud de la importancia de utilizar las cuentas adecuadas para cada caso y exista similitud entre la información que mantiene la empresa en sus archivos y lo emitido ante la Superintendencia de Compañías y Sistema de Rentas Internas.

\subsubsection{Sugerencia de Implementación de la NIC 2 para el manejo y control de los Inventarios en la Empresa Macoser S.A.}

La cuenta de Inventarios representa un activo de gran valor e importancia para la empresa, en el caso de Macoser S.A. es una cuenta que está directa y estrechamente relacionada con los resultados económicos o ventas que pueda tener durante el ejercicio económico, al tratarse de una empresa que se dedica a la compra y venta de máquinas y equipos del área textil. La Norma Internacional de Contabilidad №. 2, referida a Inventarios, se prescribe con la finalidad de emitir los lineamientos necesarios para el tratamiento contable de los inventarios, de manera que pueda mostrarse en el Estado de Situación Financiera el costo correcto de la mercadería adquirida como un activo que posteriormente representará los ingresos por las ventas que realice la empresa.

\subsubsection{Pequeño estudio del efecto tributario de la no aplicación de la NIC 2 y Deterioro}

En análisis realizado a los Estados Financieros del Año 2017, se puede observar que la utilidad gravable para el mencionado año fue de $\$ 230.212,65$, sin embargo, al aplicar la NIC 2 y realizar los ajustes correspondientes al Valor Neto Realizable y mercancía en deterioro, se obtiene una nueva utilidad gravable de $\$ 135.414,60$, disminuyendo en un $58,82 \%$ aproximadamente.

Es importante señalar que para el año 2017 la empresa obtuvo un Total de Impuesto Causado de $\$ 57.553,16$; al realizar los ajustes aplicando la NIC 2, el total de Impuesto Causado sería de $\$ 33.853,65$, disminuyendo en $\$$ $23.699,51$. Al disminuir el valor de los inventarios, como resultado de la aplicación de la NIC 2 , también disminuye la utilidad gravable y a su vez el impuesto causado para el año. La no aplicación de la NIC2 ha generado un aumento en el monto a cancelar por conceptos de tributos e impuesto para el año 2017; por otro la base de cálculo para la participación de los trabajadores en las utilidades del ejercicio es mayor, lo que incrementa el monto a cancelar por dicho concepto. 


\section{Conclusiones}

La empresa Macoser S.A. actualmente no aplica la NIC 2, por lo que se ha presentado como propuesta la aplicación de la norma, a fin de mostrar los saldos reales del inventario y llevar a cabo los ajustes necesarios para tal fin. Para realizar la propuesta se tomó como referencia los Estados Financieros de los años 2016 y 2017 de la empresa, así como también información relacionada con el stock en inventario y la mercancía de la que se dispone al 31/12/2017; partiendo de esos datos se realizó el cálculo del Valor Neto Realizable que muestra menor el valor recuperable en algunos ítems del inventario, por lo tanto amerita un ajuste en el valor de los inventarios, dando como resultado una diferencia de $\$ 31.289,05$, que se debe registrar con un cargo a la cuenta Provisión por Valor Neto de Realización, después de calcular el valor estimado para la venta menos el costo de ventas. También se debe realizar un ajuste por $\$ 63.509$, resultado de la baja que se da a maquinaria que actualmente no está apta para la venta por presentar daño y deterioro, algunos han permanecido en las bodegas por más de 5 años, y otros han sufrido deterioro, golpes, presentan fallas en el funcionamiento y la empresa no ha podido recuperarlos para la venta, por lo que se decide dar de baja a dicha mercancía. Seguidamente, se crea la provisión por pérdida en el deterioro de los inventarios, y se ajusta el valor a lo real. (Ferrari, 2018)

\section{Referencias bibliográficas}

Bohórquez, N. (07 de 2015). Implementación de norma internacional de inventarios en Colombia. Innovar, 25(57), 79 - 92.

Castillo, V. (09 de 2013). http://repositorio.unemi.edu.ec. Recuperado el 15 de 12 de 2018, de http://repositorio.unemi.edu.ec/bitstream/123456789/642/3/APLICAR\%20LOS\%2OPROCEDIMIENTOS\%20 QUE\%20LA\%2ONORMA\%20NIC\%202\%20ESTABLECE\%2C\%2OPARA\%20LA\%20EJECUCI\%C3\%93N\%20DE\%20 UN\%20SISTEMA\%20DE\%2OINVENTARIO\%20Y\%20EVALUAR\%20LOS\%20RESULTADOS\%20DE\%20FORMA\%2 0T\%С3\%89

Economiasimple. (2014). https://www.economiasimple.net/glosario/inventario. Obtenido de https://www.economiasimple.net/glosario/inventario/

Ferrari, A. (2018). ¿Qué es un sistema de administración de inventarios? Obtenido de https://www.cuidatudinero.com/13169872/que-es-un-sistema-de-administracion-de-inventarios

García, A. (2013). Almacenes, Planeación, Organización y Control. Obtenido de http://ual.dyndns.org/Biblioteca/Compras/Pdf/Unidad_07.pdf

Hansen, M. (2009). Manual para implementar Normas Internacionales de informaciòn financiera. Guayaquil: Distribuidora de Textos de Pacifico S.A.

Hernández, R., Fernández, C., \& Baptista, M. (2014). Metodologìa de la Investigaciòn. Obtenido de observatorio.epacartagena.gov.co: http://observatorio.epacartagena.gov.co/wpcontent/uploads/2017/08/metodologia-de-la-investigacion-sexta-edicion.compressed.pdf

Lòpez, Y. (2015). repositorio.uptc.edu.co. Recuperado el 10 de 12 de 2018, de https://repositorio.uptc.edu.co/bitstream/001/1949/1/TGT-708.pdf

Minango, C., \& Góngora, H. (2016). www.dspace.uce.edu.ec. Obtenido de http://www.dspace.uce.edu.ec/handle/25000/9636

NIC2. (2005). Normas Internacionales de Contabilidad Nro 2. Obtenido de http://www.normasinternacionalesdecontabilidad.es/nic/pdf/NIC02.pdf

NOSTRUM. (2015). NIC 2.- Existencia. Obtenido de http://plancontable2007.com: http://plancontable2007.com/niif-nic/nic-normas-internacionales-de-contabilidad/nic-02.html 
Palomino, L. (2016). repositorio.unsa.edu.pe. Recuperado el 23 de 10 de 2018, de http://repositorio.unsa.edu.pe/bitstream/handle/UNSA/3733/Coparilm.pdf?sequence=1\&isAllowed=y

Pérez, J. (2016). Definición.de. Obtenido de https://definicion.de/categoria/: https://definicion.de/categoria/

Pérez, J., \& Gardey, A. (2012). https://definicion.de/entrevista/. Obtenido de Deficinión.de: https://definicion.de/entrevista/

Rajadel, M., Trullàs , O., \& Simo, P. (2014). Contabilidad para todos: Introducción al registro contable. Obtenido de upcommons.upc.edu:

https://upcommons.upc.edu/bitstream/handle/2117/21190/Contabilidad+para+todos.pdf?sequence=1

Rosendo, V. (2018). Investigación de Mercados. Madrid: ESIC.

Ruiz, M. (2012). EUMED.NET. Obtenido de http://www.eumed.net/tesisdoctorales/2012/mirm/enfoque_cualitativo.html:http://www.eumed.net/tesisdoctorales/2012/mirm/enfoque_cualitativo.html

Samuel., M. (2006). Estándares/Normas Internacionales Información Financiera IFRS (NIIF). Obtenido de https://www.ecoeediciones.com/wp-content/uploads/2015/07/Estandares-Normas-internacionales-deinformaci\%C3\%B3n-financiera-IFRS-NIIF-5ta-Edici\%C3\%B3n.pdf

Sánchez Fernández, J. (s.f.). ANÁLISIS FINANCIERO. Obtenido de file:///C:/Users/DocenteUNEMI/Downloads/Jos\%C3\%A9\%20Luis\%20S\%C3\%A1nchez\%20Fern\%C3\%A1ndez \%20de\%20Valderrama_\%20Las\%20consecuencias\%20de\%20las\%20NIC_NIIF\%20para\%20el\%20An\%C3\%A 1lisis\%20Financiero.pdf

Esta obra está bajo una Licencia Creative Commons

Attribución-NoCommercial 4.0 International

\section{(cc) EY-NC}

